\title{
Pollution, bad-mouthing, and local marketing: The underground of location-based social networks
}

\author{
Helen Costa ${ }^{a}$, Luiz H.C. Merschmann ${ }^{a}$, Fabrício Barth ${ }^{\mathrm{b}}$, Fabrício Benevenuto ${ }^{\mathrm{c}, *}$ \\ ${ }^{a}$ Federal University of Ouro Preto, Ouro Preto, Brazil \\ ${ }^{\mathrm{b}}$ VAGAS Tecnologia Ltda, São Paulo, Brazil \\ ${ }^{\mathrm{c}}$ Federal University of Minas Gerais, Belo Horizonte, Brazil
}

\section{A R T I C L E I N F O}

\section{Article history:}

Received 10 September 2013

Received in revised form 20 February 2014

Accepted 26 March 2014

Available online 19 April 2014

\section{Keywords:}

Location-based social network

Social network

Tip spam

Tip analysis

\begin{abstract}
A B S T R A C T
Location Based Social Networks (LBSNs) are new Web 2.0 systems that are attracting new users in exponential rates. LBSNs like Foursquare and Yelp allow users to share their geographic location with friends through smartphones equipped with GPS, search for interesting places as well as posting tips about existing locations. By allowing users to comment on locations, LBSNs increasingly have to deal with new forms of spammers, which aim at advertising unsolicited messages on tips about locations. Spammers may jeopardize the trust of users on the system, thus, compromising its success in promoting location-based social interactions. In spite of that, the available literature is very limited in providing a deep understanding of this problem.

In this paper, we investigated the task of identifying different types of tip spam on a popular Brazilian LBSN system, namely Apontador. Based on a labeled collection of tips provided by Apontador as well as crawled information about users and locations, we identified three types of irregular tips, namely local marketing, pollution and, bad-mouthing. We leveraged our characterization study towards a classification approach able to differentiate these tips with high accuracy.
\end{abstract}

(c) 2014 Elsevier Inc. All rights reserved.

\section{Introduction}

Location Based Social Networks (LBSNs) are new Web 2.0 systems that are attracting new users in exponential rates. Nearly one in five smartphone owners access this kind of service via their mobile devices [1]. LBSNs like Foursquare and Yelp allow users to share their geographic location with friends through smartphones equipped with GPS, search for interesting places as well as posting tips about existing locations.

In Brazil, a popular LBSN system is named Apontador, ${ }^{1}$ and it includes the main features of systems like Foursquare and Yelp. It allows users to search for places, register new locations and check-in in locations using smartphones. Additionally, Apontador contains one of the most interesting features of LBSNs, which is allowing users to post-tips to existing places. Due to these tips, with a smartphone and access to a LBSN, a user might not only find nearby places to visit, but also read

\footnotetext{
* Corresponding author. Tel.: +55 3134095860.

E-mail addresses: helen.c.s.costa@gmail.com (H. Costa), luizhenrique@iceb.ufop.br (L.H.C. Merschmann), fabricio.barth@vagas.com.br (F. Barth), fabricio@dcc.ufmg.br (F. Benevenuto).

1 http://www.apontador.com.br/.
} 
suggestions about what to order, what to buy or even what to avoid on specific places. Thus, tips in LBSN work as crowdsourcing recommendations about specific locations.

Although appealing as a mechanism to enrich the user experience on the system, tips open opportunities for users to disseminate unsolicited messages. LBSNs increasingly have to deal with different forms of attacks, which aim at advertising unsolicited messages instead of true tips about locations. Most of studies in this field have focused in opinion spam $[13,14,16]$, a fake opinion that deliberately mislead readers by giving positive reviews to a place in order to promote it and/or by giving negative reviews in order to damage the place's reputation. However, little is known about the activities of other types of opportunistic behavior in the context of LBSNs. As example, consider tips containing local advertisement or even tips containing irrelevant content that is unrelated or inappropriate to the place (e.g. links to pornography websites posted at schools). This kind of tips may also jeopardize the trust of users on the existing tips available in the system, thus compromising its success in promoting location-based social interactions. Furthermore, tip spam may compromise user patience and satisfaction with the system since users need to filter out spam from what is worth reading. In spite of that, the available literature is very limited in providing a deep understanding of this problem in an environment where places are the central object.

In this paper, we address the issue of identifying different forms of tip spam in LBSNs adopting a 3-step approach. First, we categorized tip spam into three different classes based on a labelled dataset of spam and non-spam tips. Second, we analyzed a number of attributes extracted from the tips' content and from the user behavior on the system aiming at understanding their relative discriminative power to distinguish among the different classes of tip spam. Lastly, we investigated the feasibility of applying supervised machine learning methods to identify these tip spam classes. Our approach was not only able to correctly identify a significant part of the tips as spam or non-spam, but it was also able to differentiate tips from different spam classes. In this study, we identify three types of spam tips, namely (i) local marketing: tips containing local advertisement, sometimes about the current place or about a business related to it, (ii) pollution: tips with content unrelated to the place, and (iii) bad-mouthing: tips containing very aggressive comments about the places, their owners or other users who posted tips in the system.

The rest of the paper is organized as follows. Next section presents related efforts in this theme. Section 3 describes our strategy to categorize tip spam classes. Section 4 investigates a number of attributes and their ability to distinguish tips from different classes. Section 5 describes and evaluates our strategy to detect the three types of tip spam. Finally, Section 6 offers conclusions and directions for future work.

\section{Related work}

Spam detection has been observed in various social network systems, including YouTube [6], Twitter [5,11], Facebook [10], and MySpace [15]. Particularly, Benevenuto et al. [5] approached the problem of detecting spammers on Twitter. By using a labeled collection of users manually classified, they applied a classification machine learning approach to differentiate spammer users from legitimate ones. Similarly, Lee et al. [15] created social honeypots to identify a set of spammers on MySpace and Twitter. In addition to showing that social honeypots are accurate in identifying spammers, they propose a machine learning method to detect spammers in these two systems. Although these methods inspired the approach we used here, our work is complementary to them as we investigate spam in a different environment, identifying the specific features that allow us to accurately differentiate classes of tip spam. In a recent effort, Vasconcelos et al. [18] crawled Foursquare to characterize the user behavior based on information of tips, dones and toDos. Using an expectation maximization clustering algorithm, they clustered users into four groups, out of which one contained a large number of tip spammers. Thus, they presented the first evidence of spam in LBSNs.

In the context of reviews about products, Jindal and Liu [13] investigated the detection of opinion spam on product reviews, based on the analysis of reviews from amazon.com. Opinions spam are untruthful opinions that deliberately mislead readers by giving undeserving positive reviews to some target objects in order to promote the objects and/or by giving unjust or malicious negative reviews in order to damage the objects' reputation. Thus, they proposed a model to detect harmful opinions, based on duplicate reviews (copies), which inspired a few metrics proposed in our work. Recently, Kakhki et al. [14] approached the problem where users create multiple identities and use these identities to provide positive ratings on their own content or negative ratings on others' content. Then, they developed a system named Iolaus to mitigate the effect of rating manipulation in online content ratings services like tips in LBSNs. Different from these efforts, we explore other kinds of spam in LBSNs. Thus, our work is complementary to theirs.

In a previous study, we preliminarily approached the problem of detecting tip spam by creating a small test collection composed of spam and non-spam tips, and applying a binary classification strategy to detect tip spam [9]. The present work builds on this preliminary effort by providing a much more thorough, richer and solid investigation of the feasibility and tradeoffs in detecting tip spam in LBSN, considering a much larger test collection, a richer set of user attributes, as well as different classes of malicious and opportunistic behaviors. Similarly, Aggarwal et al. [2] presented a method to identify different types of spammers in Foursquare. The authors found that Foursquare users with irregular tipping activity can be classified into four categories - advertising/marketing, self-promotion, abusive or malicious. Using machine learning techniques, they were able to distinguish between legitimate and spam users on Foursquare. Our work here is complementary to [2] as we investigate a different set of features, related to content of tips, to places where the tips were posted, to users 
Table 1

Tip spam classes.

\begin{tabular}{lll}
\hline Class & Number of tips & Percentage $(\%)$ \\
\hline Local marketing & 1063 & 30.1 \\
Pollution & 1716 & 48.5 \\
Bad-mouthing & 759 & 21.4 \\
Total & 3538 & 100 \\
\hline
\end{tabular}

who posted the tips, and to the social graph. Moreover, we focus our analyses and classification approach on tips instead of users, which is a fundamentally different problem, with different applications.

\section{Tip test collection}

In order to evaluate our proposed approach to detect and differentiate classes of tip spam, we need a test collection of tips pre-classified into different classes. However, to the best of our knowledge, no such collection is publicly available for any LBSN, thus requiring us to build one. To this end we used a data collection we obtained from Apontador, a Brazilian LBSN that contains more than seven million registered places and services. Our data collection consists of two sets of data, one containing tips labelled as spam and non-spam tips, used in our previous study [9]. The other consists of data we crawled in order to enhance the features used to differentiate the classes of tips. Next, we describe both datasets.

\subsection{Labeled dataset}

Although spam presents different aspects on different environments, it is defined in most of its forms as unsolicited electronic messages, especially advertising, sent indiscriminately to users. In LBSNs, spam mostly occurs in the form of tips that aim at spreading advertisement or simply polluting the list of tips of a place. We obtained from Apontador a dataset containing tips about places manually labelled as spam or non-spam by three Apontador's moderators. They manually inspected the tips posted during the month of September, 2011 and identified 3668 tips as spam. Apontador also provided all the other tips posted during the same period.

As Apontador's manual classification relies on human judgement to decide whether a tip is spam or not, we investigate if one extra volunteer from our research group agrees with the labeling provided by Apontador's moderators. Simultaneously, we used this manual evaluation to identify different types of tip spam. In this process, we asked the volunteer to manually verify all the spam tips and classify them as spam or non-spam. The volunteer classified 130 tips as non-spam and 3538 as spam. Despite the $3.5 \%$ of tips considered as non-spam, we noted a high level of agreement with the labelled performed by Apontador, which reflects a high level of confidence of this human classification process. Thus, we considered for our study the labelled dataset obtained from Apontador without the 130 tips that we classified as non-spam.

Our second step in the labeling process consisted of asking the volunteer to provide a name that would most describe a tip category. Based on these descriptions, we could identify three classes of tip spam: local marketing, pollution, and badmouthing. Local marketing are tips containing local advertisement, sometimes about the current place or about a business related to it. Pollution contains content unrelated with the place, or irrelevant content like sentences with no apparent meaning. Finally, bad-mouthing is characterized by aggressive comments about the places containing many attacks against a place or other users. Table 1 summarizes how the labelled tips are distributed across these tip spam classes.

Aiming at using a balanced dataset, we randomly selected 3538 tips classified as non-spam. In summary, our labelled dataset contains $\mathbf{7 0 7 6}$ labelled tips equally divided as spam and non-spam. And the spam tips have a second label, being $30.1 \%$ of them classified as local marketing, $48.5 \%$ as pollution, and $21.4 \%$ as bad-mouthing. These tips were posted by $\mathbf{4 4 9 4}$ unique users to $\mathbf{5 5 8 5}$ different places.

Fig. 1 represents the sets of distinct users who have posted at least one tip classified into one of the classes we have in the dataset. We can see in the diagram that there are users who posted tips from different classes. For instance, the number of users who posted both local marketing and non-spam tips, bad mouthing and non-spam tips, pollution and non-spam tips are 23, 29, and 56, respectively, suggesting a dual behavior of a few spammers, who sometimes behave as a legitimate user. Based on this observation, we decided to focus our analyses and classification experiments on the detection of tips instead of users.

In addition to these labels, each tip obtained contains the following information: the tip content, timestamp, click counter in the link "This tip helped me", click counter in the link "Report abuse", the tip ID, the user ID, and the place ID.

\subsection{Crawling Apontador}

The labelled tips obtained from Apontador contain the unique ID of places, which allow us to gather further information about places from the Apontador API, ${ }^{2}$ useful to allow us to explore other features able to differentiate the classes of tips. Each

\footnotetext{
${ }^{2}$ http://www.api.apontador.com.br/.
} 


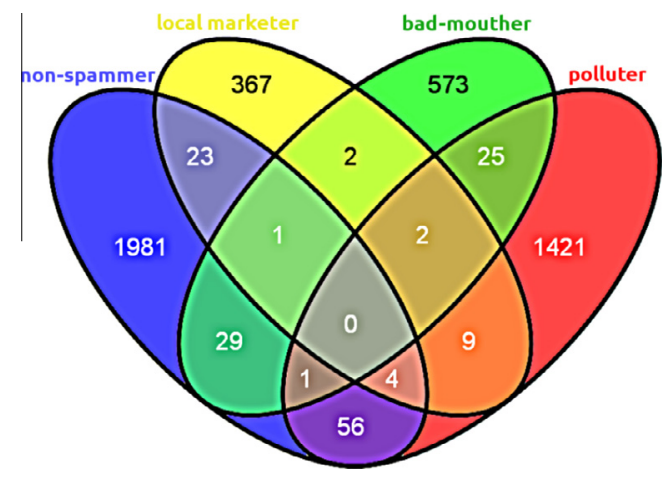

Fig. 1. Venn diagram of distinct users.

place entry on the Apontador API contains the following information: unique ID, name, description, counter click, number of tips, number of recommendations, category (e.g., restaurant, hotel, or hospital), address, phone, latitude, longitude, and information about the user who made the place registration on the system (i.e., the place owner). We developed a python crawler to gather this information for each place that appeared in the tips from the labelled dataset, which corresponds to 5585 places. ${ }^{3}$

In addition to places, we developed a second crawler that gathered information about the user's social network (list of followers and followees) as well as all the tips posted by them. By gathering the list of followers and followees of a user, new users were discovered and also gathered. We executed this process recursively until all discovered users were gathered, which corresponds to an entire weakly connected component of the Apontador graph. From the 4494 unique users that appeared in the labelled dataset we discovered and crawled a social network graph containing 137,464 users. For each crawled user we gathered the following information: user name, number of places registered, number of tips posted, and number of photos posted.

\section{Identifying behavior in LBSN}

Unlike ordinary users of LBSNs, people who spam aim at commercial intent (e.g., advertising), self-promotion, and belittlement of ideas and reputation. Assuming that users who post non-spam tips or tips containing local marketing, pollution, or bad-mouthing have different goals in the system, we expect they also differ on how they behave (e.g., what they post, to which place they post) to achieve their purposes. Thus, to verify our conjecture, in this section we analyze a large set of attributes that reflect user behavior in the system aiming at investigating their relative discriminative power to distinguish each tip class from the others. We considered four attribute sets, namely, content attributes, user attributes, place attributes, and social attributes. Next, we describe each of them and evaluate the relative ability of the identified attributes to differentiate tips of each class.

\subsection{Content attributes}

Content attributes are properties of the text of tips posted by users. The following attributes were investigated for each tip of our labelled collection: number of words, number of numeric characters (i.e., 1, 2, 3) that appear on the text, number of spam words or expressions from a popular list of spam words ${ }^{4}$ and a set of SpamAsassin rules in Portuguese ${ }^{5}$ that contains regular expressions for common spam sentences that appear on the corpus of emails, number of capital letters, number of words with all letters in capital, number of URLs, number of email addresses, number of phone numbers, and number of contact information on the text (which is the sum of number of email addresses and number of phone numbers).

Another metric we calculate is the number of offensive words on the text. To compute this metric we used a crowdsourcing list of offensive words in Portuguese. ${ }^{6}$ A second attribute related to offensive words is "Has offensive word". If the tip has at least one offensive word, the value of "Has offensive word" is 1 , otherwise, it is 0 .

We also measured the Jaccard coefficient [4] and n-gram features, defined as follows. For both metrics, we considered the set of tips associated with the user who posted the labelled tip. Let A and B be the sets of words of two tips from the same user. The Jaccard coefficient, $J(A, B)$, between $\mathrm{A}$ and $\mathrm{B}$ is given by the number of words in common in $\mathrm{A}$ and $\mathrm{B}$ divided by the total number of words in the union of both sets: $J(A, B)=|A \cap B| /|A \cup B|$. A Jaccard coefficient $J$ equal to 0 means that the two tips have no word in common, whereas J close to 1 indicates that both tips share most of the words. We also generate two

\footnotetext{
3 Apontador API is open for everyone and they suggest a limit of 15,000 req/month for developers applications. In our crawl, we did not follow the API terms of use because we signed a non-disclosure agreement (NDA) with Apontador.

${ }^{4}$ List of spam words: http://www.codex.wordpress.org/Spam_Words.

5 SpamAsassin rules to Portuguese: http://www.github.com/ppadron/spamassassin-pt-br.

${ }^{6}$ List of offensive words in Portuguese: http://www.github.com/spam-detection/badwords-pt-br.
} 

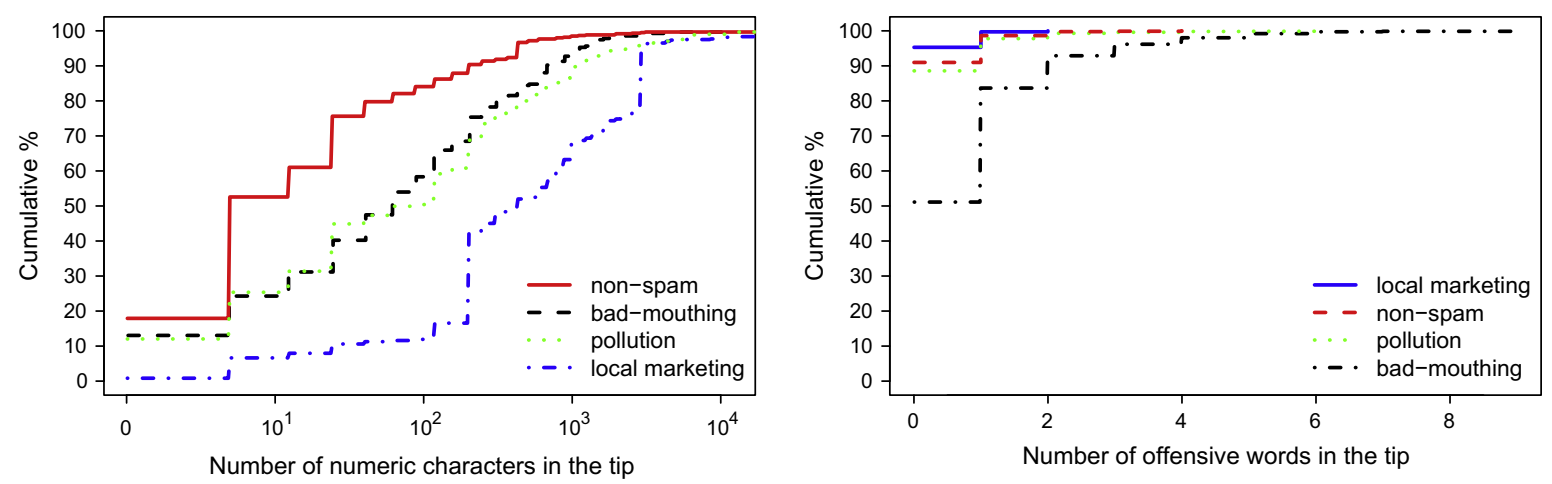

Fig. 2. Content attributes for classes of tips: number of numeric characters in the tip (left) and number of offensive words in the tip (right).

n-gram features, number of distinct n-gram and fraction of n-gram. An n-gram is a contiguous sequence of $n$ words from a given sequence of text. An n-gram of size 1 is called unigram, size 2 is brigrama and size 3 is trigram. For each tip of the user, we compute "number of distinct n-gram" as the number of distinct n-gram in the tip that were used by the user in other tips of his tip set. And to compute "fraction of n-gram", for each tip of the user, we considered the fraction of "number of distinct n-gram" per total number of distinct n-gram posted by the user in his tip set. The average of these values were used to represent the attributes and we computed the n-gram features for $n$ equals to 1,2 , and 3 .

Additionally, we compute a set of psycholinguistic features that extracts the sentiment polarity of the tip. To do this, we used a system called iFeel [3], that implements the most popular sentiment analysis methods in the literature and groups their varying scales of sentiments into two representative categories: positive affect and negative affect. The methods covered by Ifeel are: Happiness Index, SentiWordNet, SASA, PANAS-t, Emoticons, SenticNet, SentiStrength, and Combinedmethod, being the last one created by the authors to analyze the harmonic mean of the precision and recall for each previous methods and gives different weights for them. As all methods are implemented for English language, we translated our Portuguese tip collection using Google's free online language translation service. ${ }^{7}$ More details about the sentiment analysis methods can be found in [12].

Finally, we also considered two other attributes that are related to tip content, but not directly with the text. These attributes are the number of clicks on the link "This tip helped me" and the number of clicks on the link "Report abuse".

As an illustration of the potential discriminative power of the attributes we extracted from content, in Fig. 2 (left) we observe that local marketing tips have more numeric characters in the text than other tips. In fact, this happens because it is common to publish phone numbers as part of an advertise of local services. In fact, we noted that approximately $55.0 \%$ of local marketing tips have at least one contact in the text. Fig. 2 (right) shows the number of offensive words in the text of each type of tip. The plot shows that approximately $50.0 \%$ of bad-mouthing tips have at least one offensive word in the text. While for the other classes, approximately $90.0 \%$ of the tips do not have offensive words.

\subsection{User attributes}

The second set of attributes consists of specific properties of user behavior in the system. We considered the following user attributes: number of places registered by the user, number of tips posted by the user, number of photos posted by the user, the distance among all places reviewed by the user, number of different areas where the user posted a tip, tip focus of a user, and tip entropy of a user. To compute the attribute "distance among all places reviewed by the user", we measured the distance between each pair of places reviewed by the user, considering only those users who reviewed more than one different place, which correspond to $25.2 \%$ users. Otherwise, the value of this attribute is zero. Then, we computed the distance between each pair of places using the information of longitude and latitude of them. And to calculate the attribute "number of different areas where the user posted a tip", we defined an area as the geographical position of the first place reviewed by a user. If the next place reviewed by the user is within $50 \mathrm{~km}$ radius of the first area, then it belongs to the same area. Otherwise, a new area is created.

Considering the concept of area we defined, "tip focus" (TF) of a user $x$ is defined as follows:

$$
T F(x)=\frac{\max _{k} n t\left(a_{k}\right)}{t t(x)}
$$

where $n t\left(a_{k}\right)$ is the number of tips of $x$ in an area $k$ and $t t(x)$ is the total number of tips of user $x$. The TF is the highest fraction of tips that user $x$ posted in a single area over his total number of tips. In a similar way, we defined "tip entropy" (TE) of a user $x$ as follows:

\footnotetext{
7 http://www.translate.google.com/.
} 


$$
T E(x)=-\sum_{k=1}^{N} \frac{n t\left(a_{k}\right)}{t t(x)} \log _{2} \frac{n t\left(a_{k}\right)}{t t(x)}
$$

where the sum is running only over areas $(N)$ for which the number of posted tip of user $x$ is different of zero. The TE is effectively the information entropy of the distribution of tips of a user $x$ over the different areas. Thus, higher values of $T E$ denotes users whose tips are spread more uniformly across several areas, while lower $T E$ values indicate user tips more focused in less areas.

In this set of attributes we could investigate the geographical proximities of the places reviewed by the four types of users found in the dataset. To do this, we considered only tips that were posted by users who post at least two tips in Apontador. For each tip class, the percentage of local area tips: $48.6 \%$ of bad-mouthing tips, $80.8 \%$ of local marketing tips, $38.4 \%$ of pollution tips, and $54.8 \%$ of non-spam tips. A noteworthy fact here is the high percentage of local marketing tips posted in the same area. This indicates that local marketers act in certain regions and thus, targeting advertises to specific areas. On the other hand, we observed that non-spammers tend to post-tips to very distant places as well. To measure this we compute largest distance among all places in which a user posted a tip. Intuitively, one might expect that non-spammers tend to visit and post-tips only to places close to their homes or offices whereas spammers post-tips to random places. However, when we analyze the largest distance among the places a user posted a tip we noted that $60 \%$ of the non-spammers have the largest distance among tips larger than $1000 \mathrm{~km}$. In comparison with local marketers, only $20 \%$ of them have the largest distance larger than this same value. We conjecture that real users largely use LBSNs when they travel or visit different geographical regions in order to receive tips about places they are not familiar with, while local marketers and other spammers do not have the same motivation to do that. These observations suggest that user behavior extracted from geographical distance features might help to differentiate tip classes.

Another observation related to user attributes is that bad-mouthers and polluters interact less with the system tools. Fig. 3 shows that approximately $92 \%$ of the pollution tips, $83 \%$ of the bad-mouthing tips and $74 \%$ of the non-spam tips belong to users who posted up to 10 tips, whereas only $28 \%$ of local marketing tips belong to users who posted no more than this same number of tips. This indicates that, among the spammers, bad-mouthers and polluters have similar behavior and they interact less with system tools than local marketers. Another attribute that confirms this is the number of photos posted by the user. We observe that $97 \%$ of bad-mouthing and pollution tips belong to users who did not posted any photo, whereas less than $60 \%$ of local marketing tips belongs to user who did not posted any photo. We also observe that local marketers registered more places than the all other users, confirming that they have tend to interact more with the system across different features. Fig. 3 (right) shows that only 35\% of local marketing tips were posted by users who did not registered any place, whereas approximately $74 \%$ of non-spam tips and $94 \%$ of bad-mouthing and pollution tips belong to users who did not registered any place. This fact also suggests that, in addition to post-advertisements about their services, they register their business and contribute to the LBSN site.

\subsection{Place attributes}

The third set of attributes is related to the place where tips are posted. We selected 5 place attributes: number of clicks on the place page, number of tips on the place, place rating (a 5-point rating scale with 1 being the worst and 5 being the best), number of clicks on the link "Thumbs up" of the place, and number of clicks on the link "Thumbs down" of the place. Analyzing this set of attributes, we found that local marketing tips are mostly posted to places with high evaluation rating (in a five star rating system). Fig. 4 shows that more than $80 \%$ of local marketing tips are posted to places with more than 4 stars, indicating that local marketing tips usually target popular and well evaluated places. We conjecture that this behavior might be related with attempts to increase the visibility of their tips. We can also note that bad-mouthing tips tend to target bad
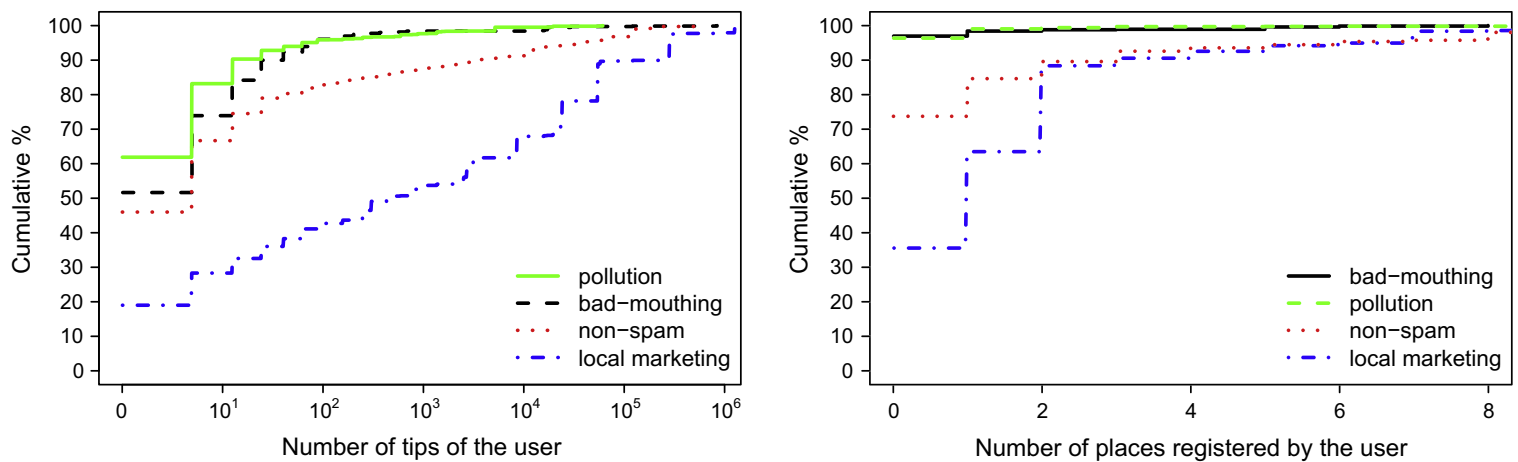

Fig. 3. User attributes for classes of tips: number of tips posted by users (left) and number of places registered by the user (right). 


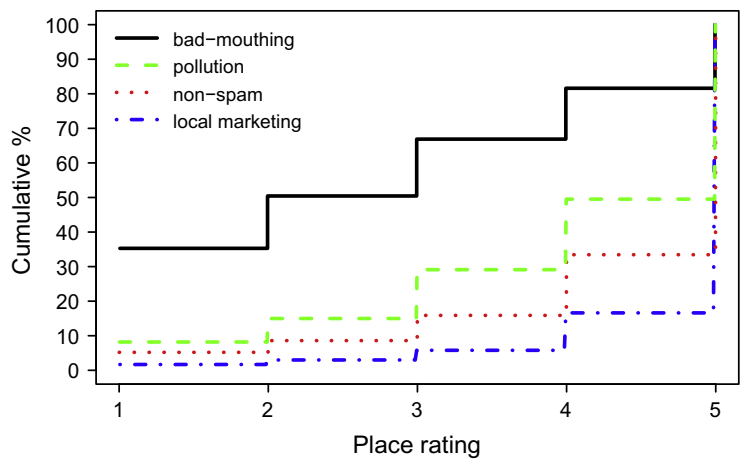

Fig. 4. 5-star rating of target places of each tip class.

quality places (i.e. almost $70 \%$ of them were posted to 1,2 or 3 stars places). This might indicate that the place they target is really a bad place or even that the bad-mouthing tips are part of an attack to decrease the rating of the place.

\subsection{Social attributes}

The fourth set of attributes captures the relationships established among users via the social network. The idea is that these attributes might capture specific interaction patterns that could help differentiating users who post non-spam tips from those who post-spam. We selected the following attributes extracted from the social network, which capture the level of (social) interaction of the corresponding user: clustering coefficient, betweenness, reciprocity, assortativity, in-degree, out-degree, degree, fraction of followers (in-degree) per followees (out-degree), and Pagerank.

The clustering coefficient of a node $i, c c(i)$, is the ratio of the number of existing edges over the number of all possible edges between $i$ 's neighbors. It measures the density of communication, not only between two users but among neighbors of neighbors. Another interesting metric to observe is the reciprocity of each user. The reciprocity $(R)$ of a user is given by $R(x)=\frac{|\operatorname{Out}(x) \cap I n(x)|}{|\operatorname{Out}(x)|}$, where Out $(x)$ is the set of users that user $x$ follows (followees) and $\operatorname{In}(x)$ is the set of users that follows user $x$ (followers). Reciprocity measures the probability of a user being followed by each user that he/she follows. Node assortativity is defined as the ratio between the node (in/out) degree and the average (in/out) degree of its neighbors. We compute node assortativity for the four types of degree-degree correlations (i.e., in-in, in-out, out-in, out-out).

We also use the Pagerank [8] algorithm on the social graph. Basically, a user has a high rank if he/she has many incoming links or the user has links coming from highly ranked users. The scores computed by the Pagerank algorithm could be used as indicators of the importance of users in terms of their participation in the LBSN.

Social attributes showed that bad-mouthers and polluters have less followers than followees. As spammers might not be interested in establishing real relationships, we can expect different patterns for spammers and non-spammers in terms of the number of incoming and outgoing links of the Apontador graph. However, instead of simple measuring the number of followers (in-degree) and followees (out-degree), we also compute the fraction of followers per followees, as shown in Fig. 5 (left). We can see that bad-mouthers and polluters have a lower ratio of followers per followees in comparison with non-spam and local marketers. Indeed, some classes of spammers may try to follow other users as an attempt to be followed back. However, most users do not follow spammers back, what produces a fraction of followers per followees smaller for bad-mouthers and polluters. Nevertheless, local marketers showed a ratio even higher than non-spammers. This confirms that this class of spammer has interacted more with the system than non-spammers.
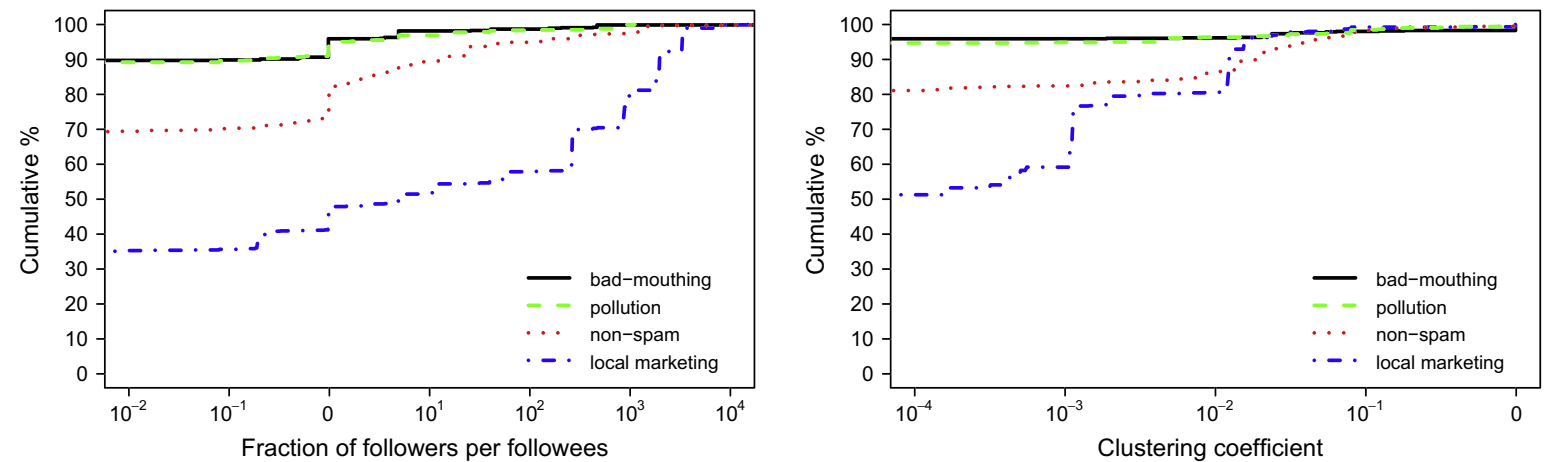

Fig. 5. Social attributes for classes of tips: followers per followees (left) and clustering coefficient (right). 
We also observed that friends of bad-mouthers and polluters are not tightly connected. The clustering coefficient measures how connected are the friends (followers and followees) of a user. Fig. 5 (right) shows the CDF for the clustering coefficient of users. As we can see local marketers and non-spammers are more tightly connected than bad-mouthers and polluters.

\subsection{Importance of the attributes}

We assessed the relative power of the 60 selected attributes in discriminating each class from the others by ranking the attributes based on the Variable Importance Measures (VIMs) from Random Forest (RF) [7] classifier, used in Section 5. The VIM used was the Mean Decrease Accuracy (MDA), which is determined during the out of bag error calculation phase. The MDA quantifies the importance of an attribute by measuring the change in prediction accuracy, when the values of the attributes are randomly permuted compared to the original observations. Table 2 summarizes the results, showing the rank of

Table 2

Attribute ranking.

\begin{tabular}{|c|c|c|}
\hline Category & MDA ranking & Description \\
\hline Content & 3 & Number of email addresses on the text \\
\hline \multirow[t]{27}{*}{32 Attributes } & 4 & Number of contact information on the text \\
\hline & 5 & Number of URLs on the text \\
\hline & 6 & Number of phone numbers on the text \\
\hline & 7 & Number of numeric characters \\
\hline & 8 & SentiStrength \\
\hline & 9 & Combined-method \\
\hline & 10 & Number of words \\
\hline & 12 & Number of distinct 1 -gram \\
\hline & 15 & Number of capital letters \\
\hline & 16 & SentiWordNet \\
\hline & 18 & SenticNet \\
\hline & $25,23,19,45,27$ & Similarity score (avg, median, max, min, sd) \\
\hline & 21 & Happiness index \\
\hline & 22 & SASA \\
\hline & 26 & Fraction of 1 -gram \\
\hline & 31 & Number of spam words and spam rules \\
\hline & 32 & Value of "Has offensive word" \\
\hline & 34 & Number of words in capital \\
\hline & 40 & Number of offensive words on the text \\
\hline & 43 & Clicks on the link "This tip helped me" \\
\hline & 50 & PANAS-t \\
\hline & 52 & Clicks on the link "Report abuse" \\
\hline & 56 & Emoticons \\
\hline & 57 & Number of distinct 3-gram \\
\hline & 58 & Fraction of 3-gram \\
\hline & 59 & Number of distinct 2-gram \\
\hline & 60 & Fraction of 2-gram \\
\hline User & 13 & Number of tips posted by the user \\
\hline \multirow[t]{6}{*}{11 attributes } & 17 & Number of photos posted by the user \\
\hline & 20 & Number of places registered by the user \\
\hline & $38,36,42,46,24$ & Distance among all places reviewed by the user (avg, median, max, min, sd) \\
\hline & 47 & Tip entropy of a user \\
\hline & 51 & Number of different areas where the user posted a tip \\
\hline & 55 & Tip focus of a user \\
\hline Place & 1 & Number of tips on the place \\
\hline \multirow[t]{4}{*}{5 attributes } & 2 & Place rating \\
\hline & 28 & Clicks on the link "Thumbs up" \\
\hline & 29 & Clicks on the link "Thumbs down" \\
\hline & 35 & Number of clicks on the place page \\
\hline Social & 11 & Fraction of followers per followees \\
\hline \multirow[t]{8}{*}{12 Attributes } & 14 & Number of followers (in-degree) \\
\hline & $44,37,41,30$ & Assortativity (in-in, in-out, out-in, out-out) \\
\hline & 33 & Degree \\
\hline & 39 & Clustering coefficient \\
\hline & 48 & Number of followees (out-degree) \\
\hline & 49 & Pagerank \\
\hline & 53 & Reciprocity \\
\hline & 54 & Betweenness \\
\hline
\end{tabular}

The 15 most discriminative attributes are in boldfaced. 
attributes from each set (content, user, place and social) according to the variable importance ranking. Note that the 15 most discriminative attributes are distributed among the four categories, which shows the importance of having investigated each one of them.

\section{Detecting classes of tip spam}

From now on, we investigate the feasibility of applying supervised learning methods in order to detect bad-mouthing, pollution and local marketing tips on Apontador. To do so, the learning algorithm copes with the attributes described in the previous section, i.e., it builds a classification model by analyzing a set of training instances (tips) represented by a vector of attribute values and a class label. In a second step, the classification model is used to classify test instances (tips) into the classes: non-spam, bad-mouthing, pollution and local marketing. In this work, we are using a training set containing prelabelled data (see Section 3). In practical scenarios, several initiatives can be used to obtain a set of labelled data (e.g., volunteers who help marking spam, professionals hired to periodically manually classify a sample of tips, etc.). Additionally, there are semi-supervised strategies in the literature that are able to obtain classification results close to supervised approaches with a much reduced labeling dataset [20]. Our goal here is to evaluate the effectiveness of supervised learning methods for the task to detect the different classes of tip spam we identified. We left as future work the effort to reduce the labeling dataset for this task.

The problem addressed here can be seen as a hierarchical classification problem, since there is a pre-defined class hierarchy as shown in Fig. 6. In this hierarchy, the first level is composed of the classes spam $(S)$ and non-spam (NS), and the second level of descendant classes of spam (bad-mouthing $(B M)$, pollution $(P L)$ and local marketing $(L M)$ ). Aiming at solving this problem, two classification approaches were considered: flat and hierarchical.

The flat multi-class classification approach is the simplest one to cope with classification problems, as it ignores the class hierarchy and perform predictions directly at the leaf nodes. Then, a unique classifier is trained to differentiate among nonspam, bad-mouthing, pollution and local marketing tips.

On the other hand, the hierarchical approach takes into account the class hierarchy by using a local information perspective. Among the different ways of using this local information, in this work we are considering a local classifier per parent node. In essence, in this approach, for each parent node in the class hierarchy, a classifier is built to distinguish among its child nodes. This approach is often coupled with top-down prediction strategy in the classification phase. Then, the classification of a new instance is processed one level at a time.

Therefore, in the problem addressed here, a root node classifier is trained to separate spam from non-spam tips, and another one, a spam node classifier, is trained to distinguish among bad-mouthing, pollution and local marketing tips (child nodes of spam). Later, during the classification phase, an instance is assigned a class of the first hierarchy level by the root node classifier. If spam is the class assigned to this instance, then the spam node classifier will assign to this instance one of the spam child classes (bad-mouthing, pollution or local marketing).

Next, the metrics used to evaluate our experimental results are presented in Section 5.1. Section 5.2 describes the classification methods adopted in this work and the experimental setup used. The results obtained from flat and hierarchical approaches are presented in Sections 5.3 and 5.4, respectively. Finally, Section 5.5 discusses the impact of reducing the attribute set on the classification effectiveness.

\subsection{Evaluation metrics}

To assess the effectiveness of our classification experiments, we adopted metrics commonly used in machine learning and information retrieval [4]. To explain these metrics in the context of our problem, we will use the confusion matrix showed in Table 3, where $a$ indicates the percentage of non-spam tips (NS) that were correctly classified, $b$ indicates the percentage of

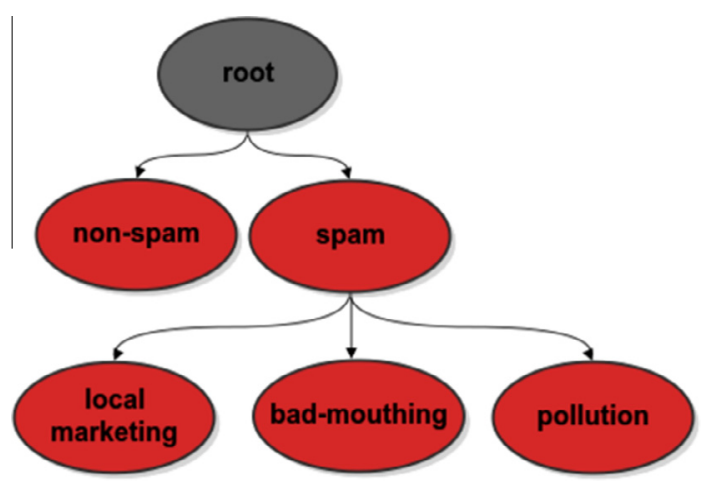

Fig. 6. Class hierarchy illustration. 
Table 3

Confusion matrix.

\begin{tabular}{|c|c|c|c|c|}
\hline \multirow[t]{2}{*}{ True label } & \multicolumn{4}{|c|}{ Predicted label } \\
\hline & NS & $L M$ & $P L$ & $B M$ \\
\hline NS & $a$ & $b$ & $c$ & $d$ \\
\hline$L M$ & $e$ & $f$ & $g$ & $h$ \\
\hline$P L$ & $i$ & $j$ & $k$ & $l$ \\
\hline$B M$ & $m$ & $n$ & 0 & $p$ \\
\hline
\end{tabular}

non-spam tips that were wrongly classified as local marketing $(L M), c$ indicates the percentage of non-spam tips that were wrongly classified as pollution $(P L)$, and $d$ indicates the percentage of non-spam tips that were wrongly classified as badmouthing $(B M)$. The same reasoning can be applied to interpret the remaining entries in this confusion matrix.

The following metrics were considered in our evaluation: recall, precision, F-measure (F1), Micro-F1 (or accuracy) and Macro-F1. The recall $(R)$ and precision $(P)$ of a class $i$ are defined as follows:

$$
R_{i}=\frac{T P_{i}}{T P_{i}+F N_{i}}, P_{i}=\frac{T P_{i}}{T P_{i}+F P_{i}},
$$

where $T P_{i}$ is the number of instances correctly assigned to class $i, F N_{i}$ is the number of instances which belong to class $i$ but are not assigned to class $i$ by the classifier, and $F P_{i}$ is the number of instances which do not belong to class $i$ but are incorrectly assigned to class $i$ by the classifier.

The values in the main diagonal of confusion matrix showed in Table 3 represent the recall of each class.

The F-measure metric is a standard way of summarizing precision and recall, and is defined as $F 1_{i}=2 \times \frac{P_{i} \times R_{i}}{P_{i}+R_{i}}$. The F-measure metric reaches its best value at 1 (indicating a perfect prediction) and worst at 0 . The overall F-measure value of the entire classification problem can be calculated in two different ways: Micro-F1 and Macro-F1.

The Micro-F1 is computed from the global precision and recall values calculated for all classes as follows:

$$
R=\frac{\sum_{i=1}^{m} T P_{i}}{\sum_{i=1}^{m}\left(T P_{i}+F N_{i}\right)}, P=\frac{\sum_{i=1}^{m} T P_{i}}{\sum_{i=1}^{m}\left(T P_{i}+F P_{i}\right)},
$$

where $m$ is the number of classes. The Micro-F1 is then defined as Micro-F1 $=2 \times \frac{P \times R}{P+R}$. Basically, this metric gives equal importance to each tip, independently of its class, and therefore, it tends to be dominated by classifier's performance on majority classes.

In a different way, Macro-F1 gives equal importance to each class, regardless of its relative size. Then, it is more affected by classifier's performance on minority classes. Macro-F1 is obtained by computing the average of F-measure values of each class as Macro-F1 $=\frac{\sum_{i=1}^{m} F 1_{i}}{m}$, where $m$ is the number of classes.

\subsection{The classifiers and experimental setup}

In this work, experiments were conducted using Support Vector Machine (SVM) [17] and Random Forest (RF) [7] classifiers, which are state-of-art techniques in classification. For all the classification approaches, both SVM and RF classifiers were evaluated.

Considering that the set of training instances are represented as points in space, the basic idea of the Support Vector Machine is to find an optimal separating hyperplane, i.e., a decision boundary that separates with a maximum margin the training data into two portions of an $\mathrm{N}$-dimensional space. Then, given a new instance to be classified, it is mapped into that same space and predicted to belong to a class based on its position in relation to the separating hyperplane. To deal with non-linearly separable data, SVM transforms the original training data into a higher dimension using a nonlinear mapping. Once the data have been transformed into a new higher dimensional space, SVM searches for a linear separating hyperplane in this new space. Although this method has been originally designed for binary classification, different extensions were proposed in the literature to make it suitable for multi-class problems.

The implementation of SVM used in our experiments is provided by Weka [19], a free and open source software widely used for data mining. We used the Radial Basis Function (RBF) kernel to allow SVM models to perform separations even with very complex boundaries. Aiming at finding the best parameters setting for the dataset used in this work, a tuning process has been run using the GridSearch algorithm also implemented in the Weka tool. As result of this tuning process, for the flat classification approach, in which a unique classifier is trained, the parameter values $c=1024$ and gamma $=0.0625$ were adopted in our experiments. As for hierarchical approach, since two classifiers are built, we obtained two different sets of parameter values: $c=512$ and gamma $=0.125$ for the classifier trained to distinguish between spam and non-spam, and $c=80$ and gamma $=0.5$ for the classifier used to identify bad-mouthing, pollution and local marketing tips.

Random Forest is an ensemble classifier that builds many decision trees (forest) and outputs the class having most votes over all the trees in the forest. Each decision tree is grown from a random subset of the training dataset. In addition, during the tree growing process, a random subset of the available attributes is used to determine the best split for each node of the 
tree. For classifying a new instance (tip), it is pushed down each of the trees in the forest. Then, each tree gives a classification, i.e., a class receives one vote of that tree. The Random Forest prediction is the class having the most votes.

The experiments involving RF were carried out using the algorithm implemented in the Weka software. In order to search for the best parameter set for the given training dataset, a grid search parameter optimization algorithm (GridSearch) was run on parameters numFeatures (used in random selection of attributes) and numTrees (number of trees to be generated) of the classifier. As result, for the flat classification approach, the parameter values numFeatures $=24$ and numTrees $=275$ were adopted in our experiments. As for hierarchical approach, the two different sets of parameter values are: numFeatures $=11$ and numTrees $=155$ for the classifier trained to distinguish between spam and non-spam, and numFeatures $=22$ and numTrees $=165$ for the classifier used to identify bad-mouthing, pollution and local marketing tips.

The predictive performance was measured using a $10 \times 5$-fold cross-validation method $(10 \times 5-C V)$. In each $5-C V$ test, the original dataset is partitioned into 5 exclusive sets, out of which four are used as training data, and the remaining one is used for testing the classifier. The classification process is then repeated 5 times, with each of the 5 sets used exactly once as the test data, thus producing 5 results. The entire $5-C V$ was repeated 10 times with different seeds used to shuffle the original dataset, thus producing 50 potentially different results. So, the results reported in Sections 5.3 and 5.4 are averages of the 50 runs. With $95 \%$ of confidence, the results reported in Sections 5.3 and 5.4 do not differ from the average in more than $2 \%$.

\subsection{Flat classification}

As previously mentioned, in the flat classification approach, a unique classifier is trained from a training dataset containing instances associated with the classes non-spam, bad-mouthing, pollution and local marketing. Then, given a new instance to be classified, the classifier will assign to it one of these training classes.

Table 4 shows the confusion matrix obtained as result of our experiments with the flat classification approach using the SVM classifier. Each presented value corresponds to the percentage of $X$ tips that were classified as $Y$ tip, where $X$ and $Y$ are the types of tips: non-spam (NS), local marketing $(L M)$, pollution $(P L)$ and bad-mouthing $(B M)$. The boldfaced values in this matrix indicate the recall of the classes. As can be seen, $93.5 \%$ of non-spam, $74.6 \%$ of local marketing, $60.7 \%$ of pollution and $48.4 \%$ of bad-mouthing were correctly classified by SVM. Despite the good results achieved for the classes non-spam and local marketing (recall $>74 \%$ ), a significant fraction of pollution and bad-mouthing tips were misclassified. Among these misclassification errors we have $28.2 \%$ of pollution and $19.3 \%$ of bad-mouthing that were incorrectly classified as non-spam. Moreover, bad-mouthing tips were erroneously classified as pollution in $30.4 \%$ of cases. As a summary of the classification results with SVM, Micro-F1 value is $77.9 \%$, which means that the classifier is predicting the correct class for almost $78 \%$ of the tips. The per-class F1 values are $87.8 \%, 81.1 \%, 62.9 \%$ and $54.3 \%$, for non-spam, local marketing, pollution and badmouthing classes, respectively, resulting in a Macro-F1 equal to $71.5 \%$.

Table 5 shows the confusion matrix obtained as result of our experiments with the flat classification approach using Random Forest classifier. The recall of the classes are in bold font and indicate that $95.7 \%$ of non-spam, $77.6 \%$ of local marketing, $65.2 \%$ of pollution and $54.2 \%$ of bad-mouthing were correctly classified by Random Forest. Again, despite the good results achieved for the classes non-spam and local marketing (recall $>77 \%$ ), a significant fraction of pollution and bad-mouthing tips were misclassified. However, when compared with the results achieved by SVM classifier, we can note that the Random Forest classifier obtained the best recall values for all classes evaluated (non-spam, local marketing, pollution and badmouthing). In addition, regarding to Micro-F1 and Macro-F1 metrics, the Random Forest also outperformed the SVM classifier. It has achieved Micro-F1 $=81.2 \%$ and Macro-F1 $=75.5 \%$, which was computed from the following per-class F1 values: 90.2\% (for non-spam), $83.1 \%$ (for local marketing), $67.8 \%$ (for pollution) and $60.7 \%$ (for bad-mouthing).

\subsection{Hierarchical classification}

Although Random Forest has achieved the best results using the flat classification approach, we decided to evaluate both, Random Forest and SVM, in a hierarchical strategy because as the approaches are very different and, thus, the performance of the classifiers can be different as well.

As explained in Section 5, we are considering a local classifier per parent node in the hierarchical approach. It means that, in the problem addressed here, two classifiers are trained. The first one is used to separate the classes of the first hierarchy

Table 4

SVM flat classification.

\begin{tabular}{lcccc}
\hline True label & Predicted label & & & \\
\cline { 2 - 5 } & NS $(\%)$ & $L M(\%)$ & $P L(\%)$ & $B M(\%)$ \\
\hline$N S$ & $\mathbf{9 3 . 5}$ & 0.6 & 3.6 & 2.3 \\
$L M$ & 5.3 & $\mathbf{7 4 . 6}$ & 18.9 & 1.2 \\
$P L$ & 3.3 & $\mathbf{6 0 . 7}$ & 7.6 \\
$B M$ & 28.2 & 1.9 & 30.4 & $\mathbf{4 8 . 4}$ \\
\hline
\end{tabular}


Table 5

Random Forest flat classification.

\begin{tabular}{lcccc}
\hline True label & Predicted label & & & \\
\cline { 2 - 5 } & $N S(\%)$ & $L M(\%)$ & $P L(\%)$ & $B M(\%)$ \\
\hline$N S$ & $\mathbf{9 5 . 7}$ & 0.5 & 2.4 & 1.4 \\
$L M$ & 5.3 & $\mathbf{7 7 . 6}$ & 16.6 & 0.5 \\
$P L$ & 23.3 & 4.1 & $\mathbf{6 5 . 2}$ & 7.4 \\
$B M$ & 17.2 & 1.4 & 27.2 & $\mathbf{5 4 . 2}$ \\
\hline
\end{tabular}

Table 6

SVM hierarchical classification (first classification phase).

\begin{tabular}{llr}
\hline True label & \multicolumn{2}{l}{ Predicted label } \\
\cline { 2 - 3 } & NS (\%) & $S(\%)$ \\
\hline$N S$ & $\mathbf{9 1 . 5}$ & 8.5 \\
$S$ & 16.1 & $\mathbf{8 3 . 9}$ \\
\hline
\end{tabular}

Table 7

SVM hierarchical classification (second classification phase).

\begin{tabular}{lllc}
\hline \multirow{2}{*}{ True label } & \multicolumn{2}{l}{ Predicted label } & \\
\cline { 2 - 4 } & $L M(\%)$ & $P L(\%)$ & $B M(\%)$ \\
\hline$L M$ & $\mathbf{7 8 . 2}$ & 21.2 & 0.6 \\
$P L$ & 4.6 & $\mathbf{8 5 . 7}$ & 9.7 \\
$B M$ & 1.4 & 40.0 & $\mathbf{5 8 . 6}$ \\
\hline
\end{tabular}

Table 8

SVM final hierarchical classification results.

\begin{tabular}{llccc}
\hline True label & Predicted label & & & \\
\cline { 2 - 5 } & $N S(\%)$ & $L M(\%)$ & $P L(\%)$ & $B M(\%)$ \\
\hline$N S$ & $\mathbf{9 1 . 7}$ & 0.8 & 5.0 & 2.5 \\
$L M$ & 4.7 & $\mathbf{7 4 . 7}$ & 19.7 & 0.9 \\
$P L$ & 3.5 & $\mathbf{6 4 . 9}$ & 7.4 \\
$B M$ & 24.2 & 1.4 & 34.4 & $\mathbf{5 0 . 3}$ \\
\hline
\end{tabular}

level (non-spam $(N S)$ and spam $(S)$ ). It is trained from a training dataset which contains tips labelled as non-spam or spam. The second classifier is constructed to distinguish among local marketing $(L M)$, pollution $(P L)$ and bad-mouthing $(B M)$ tips (spam child classes). In this way, it is trained from the same training dataset used by the first classifier excluding the non-spam tips and detailing the spam tips into local marketing, pollution and bad-mouthing.

Tables 6 and 7 show the confusion matrix obtained as the result of the first phase and the second phase of the hierarchical classification approach using SVM, respectively.

The SVM final result of these two classification phases is aggregated in the confusion matrix presented in Table 8. In addition, the SVM classifier has achieved Micro-F1 $=78.4 \%$ and Macro-F1 $=72.3 \%$ (computed from the following per-class $\mathrm{F} 1$ values: $88.4 \%$ for non-spam, $81.2 \%$ for local marketing, $63.9 \%$ for pollution and $55.8 \%$ for bad-mouthing).

Similarly, Tables 9-11 represent the results of the first phase, second phase and aggregated result of these two phases for the hierarchical classification approach using Random Forest, respectively. Again, when compared with the results achieved by SVM classifier, we can see that the Random Forest classifier obtained the best recall values for all classes evaluated.

Furthermore, when comparing Random Forest hierarchical classification results to the best flat classification results presented in Table 5, we verify that, regarding to recall, despite the worse result achieved for the class non-spam, the hierarchical approach provided better results for the classes pollution and bad-mouthing, which were the classes with the worst performance in all experiments. However, evaluating the global classification metrics, we can observe that the best results for flat classification approach (Micro-F1 $=81.2 \%$ and Macro-F1 $=75.5 \%$ ) and the results for Random Forest hierarchical classification approach (Micro-F1 $=81.2 \%$ and Macro-F1 $=75.7 \%$, computed from the following per-class F1 values: $90.7 \%$ for non-spam, $82.7 \%$ for local marketing, $68.2 \%$ for pollution and $61.2 \%$ for bad-mouthing) are almost the same. Thus, choosing an approach will depend only on what classes is desirable to prioritize in the performance of the classifier. 
Table 9

Random Forest hierarchical classification (first classification phase)

\begin{tabular}{llr}
\hline True label & \multicolumn{2}{l}{ Predicted label } \\
\cline { 2 - 3 } & NS (\%) & $S(\%)$ \\
\hline NS & $\mathbf{9 3 . 8}$ & 6.2 \\
$S$ & 13.2 & $\mathbf{8 6 . 8}$ \\
\hline
\end{tabular}

Table 10

Random Forest hierarchical classification (second classification phase).

\begin{tabular}{lllc}
\hline True label & \multicolumn{3}{l}{ Predicted label } \\
\cline { 2 - 4 } & $L M(\%)$ & $P L(\%)$ & $B M(\%)$ \\
\hline$L M$ & $\mathbf{8 1 . 1}$ & 18.0 & 0.9 \\
$P L$ & 4.7 & $\mathbf{8 5 . 2}$ & 10.1 \\
$B M$ & 1.5 & 35.8 & $\mathbf{6 2 . 7}$ \\
\hline
\end{tabular}

Table 11

Random Forest final hierarchical classification results.

\begin{tabular}{lcccc}
\hline \multirow{2}{*}{ True label } & Predicted label & & & \\
\cline { 2 - 5 } & NS $(\%)$ & $L M(\%)$ & $P L(\%)$ & $B M(\%)$ \\
\hline$N S$ & $\mathbf{9 3 . 1}$ & 0.6 & 4.2 & 2.1 \\
$L M$ & 5.7 & $\mathbf{7 6 . 4}$ & 17.0 & 0.9 \\
$P L$ & 3.8 & $\mathbf{6 8 . 8}$ & 8.2 \\
$B M$ & 19.2 & 1.1 & 32.4 & $\mathbf{5 6 . 6}$ \\
\hline
\end{tabular}
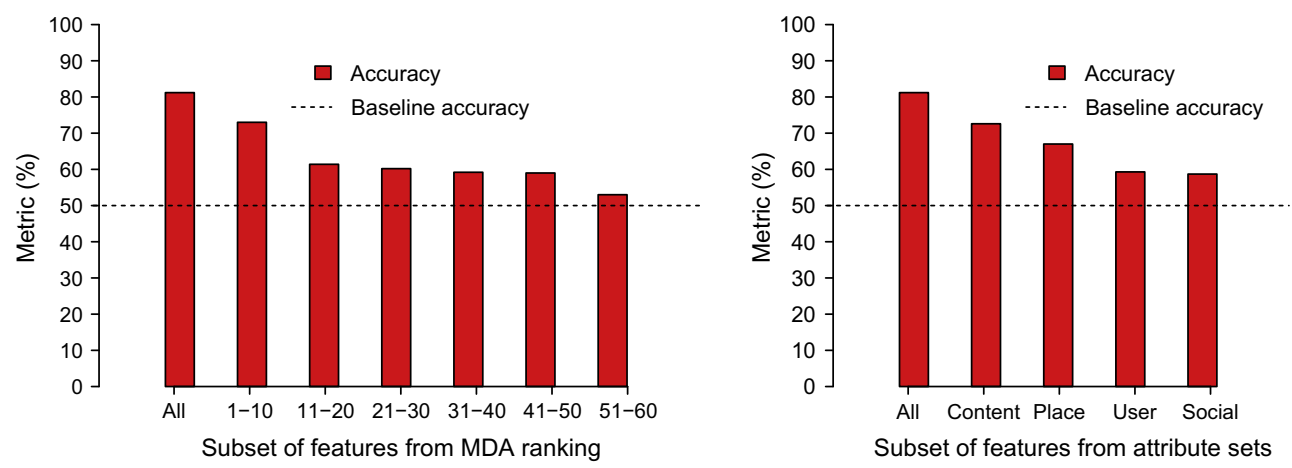

Fig. 7. Results with subsets of attributes.

\subsection{Impact of reducing the attribute set}

In Section 4.5 we evaluated the relative power of the attributes considered in our dataset in discriminating each tip class from the others. However, as important as to understand the relevance of these attributes, is to assess whether competitive classification performance can be reached with fewer attributes and different sets of attributes. This type of analysis is important for the following reasons. First, since it is expected that the spammers will evolve and adapt their strategies to fool anti-spam systems, in the course of time, some attributes may become less important while others may gain importance. Second, given the huge dimensions of the datasets related to social network applications, reaching accurate classification results from reduced datasets is desirable to speed up the classification process and to improve the model's interpretability.

In order to evaluate the classifier performance considering different subsets of attributes, we conducted experiments using subsets of 10 attributes that occupy contiguous positions in the ranking (i.e., the first top 10 attributes, the next 10 attributes and so on) presented in Table 2 . The plots show the accuracy value for all attributes, for different subsets of attributes and for a baseline classifier that considers all tips as non-spam. We also conducted experiments using subsets according to each set of attributes (content, user, place and social). Fig. 7 shows the results of these experiments. We can note that our classification provides gains over the baseline for all subsets of attributes evaluated, i.e., even low ranked attributes have 
some discriminatory power. In addition, significant improvements over the baseline can be reached even if only a set of attributes (e.g., content attributes) considered in our experiments can be obtained. This observations are important if we consider the high cost for computing some of the social attributes. We can see that if some attributes cannot be obtained, we are still able to obtain significant improvements over a baseline approach.

\section{Concluding remarks}

In this paper we approached the problem of detecting different kinds of tip spam in a popular Brazilian location-based social network. We crawled and manually analyzed a dataset of tips posted on Apontador, to label them as non-spam and spam tips, which are further labelled into three spam subclasses: local marketing, bad-mouthing, and pollution. In order to identify attributes able to distinguish these classes, we crawled the Apontador site to obtain information of places, users and the social graph of more than 137,000 users. Based on this dataset, we analyze the characteristics and behavior of different types of users who are posting tip spam and we did a characterization of the tips of this labelled collection, unveiling several behavioral aspects of LBSNs users and their tip's content that are able to differentiate tip classes. We then leverage our findings towards a supervised classification technique able to effectively distinguish among non-spam, local marketing, bad-mouthing, and pollution tips. Particularly, our flat classification approach was able to detect correctly almost $78 \%$ of the local marketing tips, $65 \%$ of pollution tips, $54 \%$ of bad-mouthing tips, wrongly classifying only about $4 \%$ of non-spam tips. Thus, our proposed approach poses a promising alternative to simply considering all tips as non-spam or to randomly selecting tips for manual inspection. We also investigated a hierarchical version of the proposed approach, which provided even better results for identifying the classes of pollution and bad-mouthing tips, which are the classes with the worst performance in all experiments. Finally, our experimental results show that even with a small subset of attributes (containing 10 attributes), our classification approach was able to reach high accuracy (73\%). And our classification could produce significant benefits even when we used only one set of attributes, being the best performance for content attributes, with an accuracy of almost $73 \%$.

We hope that the identification, characterization, differentiation of spam classes in LBSNs presented here may also have implications to other review-based systems and could be combined with other defence strategies. As example, we noted that bad-mouthing tips are posted to low rated places. Thus, after detecting bad-mouthing tips, one could try to differentiate if they are real tips from users that disliked the place or if they are related to a rating collusion attack. This could be done by using a rating defence mechanism, as Iolaus, proposed in [14].

Another important possibility that our approach unveils is related to the local marketing tips. We noted that local marketers are active users that create places, thus, contributing positively to the system in some aspects. By identifying them, the LBSN system could potentially offer them an agreement for advertising their services in certain places as "sponsored tips" instead of removing their tips from a place or even banishing them from the system.

As our final contribution, we make our labelled collection of tip spam available for the research community.

\section{Acknowledgements}

This research is partially funded by the Brazilian National Institute of Science and Technology for the Web (MCT/CNPq/ INCT Grant No. 573871/2008-6), and by the authors' individual grants from CNPq, CAPES and FAPEMIG. The authors also would like to thank Apontador for the data provided, which made this research possible.

\section{References}

[1] Nearly 1 in 5 smartphone owners access check-in services via their mobile device. <http://bit.ly/mgaCIG>

[2] A. Aggarwal, J. Almeida, P. Kumaraguru, Detection of spam tipping behaviour on foursquare, in: Proceedings of the 22Nd International Conference on World Wide Web Companion, WWW'13 Companion, 2013, pp. 641-648.

[3] M. Araújo, P. Gonçalves, F. Benevenuto, M. Cha, ifeel: a system that compares and combines sentiment analysis methods, in: WWW (Companion Volume), International World Wide Web Conference (WWW'14), 2014.

[4] R. Baeza-Yates, B. Ribeiro-Neto, Modern Information Retrieval, ACM Press/Addison-Wesley, 1999.

[5] F. Benevenuto, G. Magno, T. Rodrigues, V. Almeida, Detecting spammers on twitter, in: 7th Annual Collaboration, Electronic Messaging, Anti-Abuse and Spam Conference (CEAS), 2010.

[6] F. Benevenuto, T. Rodrigues, V. Almeida, J. Almeida, M. Gonçalves, Detecting spammers and content promoters in online video social networks, in: Int'l ACM Conference on Research and Development in Information Retrieval (SIGIR), 2009, pp. 620-627.

[7] L. Breiman, Random forests, Mach. Learn. 45 (1) (2001) 5-32.

[8] S. Brin, L. Page, The anatomy of a large-scale hypertextual web search engine, Comput. Netw. ISDN Syst. 30 (1-7) (1998) 107-117.

[9] H. Costa, F. Benevenuto, L.H. de Campos Merschmann, Detecting tip spam in location-based social networks, in: Proceedings of the 28th Annual ACM Symposium on Applied Computing (SAC), 2013, pp. 724-729.

[10] H. Gao, J. Hu, C. Wilson, Z. Li, Y. Chen, B.Y. Zhao, Detecting and characterizing social spam campaigns, in: ACM Int'l Conference on Internet Measurement (IMC), pp. 35-47

[11] S. Ghosh, B. Viswanath, F. Kooti, N.K. Sharma, K. Gautam, F. Benevenuto, N. Ganguly, K. Gummadi, Understanding and combating link farming in the twitter social network, in: Int'l World Wide Web Conference (WWW'12), 2012, pp. 61-70.

[12] P. Gonçalves, M. Araújo, F. Benevenuto, M. Cha, Comparing and combining sentiment analysis methods, in: Proceedings of the First ACM Conference on Online Social Networks, COSN '13, 2013, pp. 27-38.

[13] N. Jindal, B. Liu, Opinion spam and analysis, in: ACM International Conference of Web Search and Data Mining (WSDM), 2008 , pp. 219-230.

[14] A.M. Kakhki, C. Kliman-Silver, A. Mislove, Iolaus: securing online content rating systems, in: Int'l World Wide Web Conference (WWW'13), 2013, pp. 919-930. 
[15] K. Lee, J. Caverlee, S. Webb, Uncovering social spammers: social honeypots + machine learning, in: ACM Int'l Conference on Research and Development in Information Retrieval (SIGIR), 2010, pp. 435-442.

[16] M. Ott, Y. Choi, C. Cardie, J.T. Hancock, Finding deceptive opinion spam by any stretch of the imagination, in: Proceedings of the 49th Annual Meeting of the Association for Computational Linguistics: Human Language Technologies, vol. 1, HLT '11, 2011, pp. 309-319.

[17] I. Tsochantaridis, T. Joachims, T. Hofmann, Y. Altun, Large margin methods for structured and interdependent output variables, J. Mach. Learn. Res. (JMLR) 6 (2005) 1453-1484.

[18] M. Vasconcelos, S. Ricci, J. Almeida, F. Benevenuto, V. Almeida, Tips, dones and to-dos: uncovering user profiles in foursquare, in: ACM Int'l Conference of Web Search and Data Mining (WSDM), 2012, pp. 653-662.

[19] I. Witten, E. Frank, Data Mining: Practical Machine Learning Tools and Techniques, Morgan Kaufman, 2005.

[20] X. Zhu, Semi-supervised learning literature survey, Tech. Rep. 1530, Computer Sciences, University of Wisconsin-Madison, 2005. 